\title{
Proposal of a modified transcervical endometrial resection (TCER) technique for menorrhagia treatment. Feasibility, efficacy, and patients' acceptability
}

\author{
Pietro Litta • Luigi Nappi • Pasquale Florio • \\ Luca Mencaglia • Mario Franchini • Stefano Angioni
}

Received: 11 January 2014 / Accepted: 16 April 2014 / Published online: 30 April 2014

(C) Springer-Verlag Berlin Heidelberg 2014

\begin{abstract}
The aim of this study is to evaluate the feasibility, efficacy, safeness, and patients' acceptability of a modified transcervical endometrial resection (TCER) technique for the treatment of menorrhagia. Eighty-four premenopausal women with menorrhagia after careful investigation and 2 months therapy with GnRHa underwent a modified TCER. It was performed with a standard dual channel, 26 French irrigating resectoscope (Karl Storz, GmbH, Germany) after cervix dilatation to $10 \mathrm{~mm}$ and sorbitol mannitol solution used as distension medium. The modified technique was based on the resection of the endometrium and of the first myometrial layers only on the anterior and posterior walls, without treating fundus and cornual areas as usually performed. Endometrial resection was performed to a depth of 4 to $5 \mathrm{~mm}$. Clinical and hysteroscopic follow-up was performed
\end{abstract}

\section{P. Litta}

Department of Gynecological Science and Human Reproduction,

University of Padua, Padua, Italy

\section{Nappi}

Department of Medical and Surgical Sciences, Institute of Obstetrics and Gynaecology, University of Foggia, Foggia, Italy

P. Florio

U.O.C. Obstetrics \& Gynecology, "San Giuseppe" Hospital, Empoli, Italy

\section{Mencaglia}

Section of Gynecology, Centro Oncologico Fiorentino, Sesto Fiorentino, Italy

M. Franchini

Palagi Freestanding Unit, Florence, Italy

\section{S. Angioni $(\bowtie)$}

Department of Surgical Sciences, Section of Obstetrics \& Gynecology, University of Cagliari, Azienda Ospedaliero Universitaria, Blocco Q, SS554, Monserrato, Cagliari, Italy e-mail: sangioni@yahoo.it for 60 months. Early and late complications, changing in bleeding patterns, and patients' satisfaction were recorded. Sixty-four out of 73 patients that completed the 60 months improved. Eumenorrhea was achieved in $68.5 \%$, hypomenorrhea in $5.5 \%$, and amenorrhea in $13.7 \%$. Most of the patients (86.3\%) showed satisfaction at the follow-up interview. Control hysteroscopy showed that post modified TCER uterine cavity maintained the possibility of macroscopic and histopathology investigation during follow-up. Modified TCER is a technique easy to perform and effective in the long-term resolution of menorrhagia. In particular, it avoids the formation of synechiae and the shrinkage of the uterine cavity that may be the cause of various long-term complications, such as the delay in the diagnosis of endometrial carcinoma onset.

Keywords Menorrhagia · AUB · Endometrial resection . Minimally invasive surgery $\cdot$ Hysteroscopy

\section{Introduction}

Hysteroscopic transcervical endometrial resection (TCER) is a minimally invasive surgical technique developed in recent years with the purpose of removing the entire thickness of the endometrium lining of the uterus [1]. Indeed, to suppress menstruation successfully, it is essential to remove the full thickness of this lining together with the superficial myometrium, including the deep endometrial basal glands which are believed to be the primary foci for endometrial regrowth $[1,2]$. However, TCER is not always completely successful and, in some cases, additional surgical treatment is required, thus limiting the benefits related to the reduced trauma and post-operative complications to the woman. The risk of failure and the expense of multiple treatments opened a debate whether endometrial ablation should replace or not 
hysterectomy [3], or if it might be an effective therapy for women with hyperplasia, with abnormal uterine bleeding, with high risk for medical therapy or hysterectomy [4]. The emerging clinical opinion is that TCER is an effective and safe alternative to hysterectomy that should be offered to women with menorrhagia for the relief of their heavy menstrual bleeding, together with the caution that there should be the possibility of further surgery, either repeat endometrial ablation or hysterectomy [5].

Matter of discussion related to TCER is also the putative occurrence of other problems related to the endometrial injury, as in the case of immediate (vascular or metabolic type complications (fluid overload) and perforation), or delayed complications (as in the case of the development of partial intrauterine dense adhesions and/or total obliteration of the cavity) [5-12]. Therefore, the ideal method of TCER associating high efficacy to nice tolerability and low incidence of complications is still far from being achieved. In the present study, we evaluated short- and long-term outcomes associated with a new TCER technique to treat menorrhagia that differ from the standard one in the fact that uterine fundus and cornual areas are not removed in the modified technique.

\section{Materials and methods}

\section{Subjects}

For this prospective cohort study, we consecutively enrolled from October 2, 2000 to September 24, 2005 all women suffering of menorrhagia who referred to our tertiary centers of women health care. The diagnosis of menorrhagia was performed by means of a pictorial blood loss assessment chart, adjusted to our needs in patients describing a history of heavy menstrual blood loss over several consecutive cycles [13]. A scoring system ranging from 1 to 10 was used, with $1=$ slightly soiled tampon, $5=$ moderately soiled, and $10=$ heavily soiled. Sanitary napkins were assigned ascending scores from 1 to 20 . A total score more than 100 for each pictorial chart was meant as a confirmed diagnosis of menorrhagia [14]. We considered for the study only patients who had performed a full clinical evaluation including colposcopy and Papanicolaou test, transvaginal ultrasonography, and hysteroscopy with endometrial biopsy [15]. Exclusion criteria were the following: not confirmed diagnosis of menorrhagia, uterine size $>12 \mathrm{~cm}$, presence of large organic intrauterine lesions (endometrial polyp $>3 \mathrm{~cm}$, submucous myomas G0 $>2 \mathrm{~cm}$ or submucous myomas G1 and G2); desire of future pregnancy, cervical and endometrial pre- and malignant conditions or adnexal pathologies; and debilitating medical condition. Any medical hormonal treatment was suspended at least 1 month before enrollment. All procedures followed were in accordance with the ethical standards of the responsible committee on human experimentation (institutional and national) and with the Helsinki Declaration of 1975, as revised in 2000. Informed consent was obtained from all patients for being included in the study.

\section{Surgical procedure: modified TCER}

All the women underwent therapy with GnRH analogs (Leuprolide Acetate $3.75 \mathrm{mg}$ ) for 2 months (every 28 days) before surgery, as in standard practice [6]. All procedures were performed under general anesthesia, with induction by propofol $2 \mathrm{mg} \mathrm{kg}^{-1}$ and spontaneous ventilation with a mixture of $60 \%$ nitrous oxide and $40 \%$ oxygen isofluothane, or spinal anesthesia in selected patient [16]. Hysteroscopic MTCER was performed with a standard dual channel, 26 French irrigating resectoscope (Karl Storz, GmbH, Germany) after that cervix was dilated to $10 \mathrm{~mm}$. The uterine cavity was distended with sorbitol mannitol solution used as distension medium, and a suction-irrigating unit (Endomat, Karl Storz, GmbH, Germany) was used to provide positive pressure $(120 \mathrm{mmHg})$ and continuous outflow suction control ( 0.5 bar). Fluid balance was carefully monitored throughout the procedure that was interrupted if fluid deficit was over $1,000 \mathrm{~cm}^{3}$. Surgical time was recorded starting at resectoscope introduction inside the uterus and ending at its last removal.

Compared to the technique described by Wortman and Dagget [2], our modified TCER began on either anterior or posterior uterine wall and was based on the resection of the anterior cardinal strip of tissue followed by resection of the posterior and the two lateral cardinal strips without treating fundus and cornual areas (Fig. 1). The conventional TCER approach consists in the treatment of fundal and cornual areas by the equatorial loop and/or the use of the rollerball electrode. Endometrial resection was performed to a depth of 4 to $5 \mathrm{~mm}$, and endomyometrial strips were removed from the cavity and sent for histological assessment. The procedure was scheduled for a 1-day surgery.

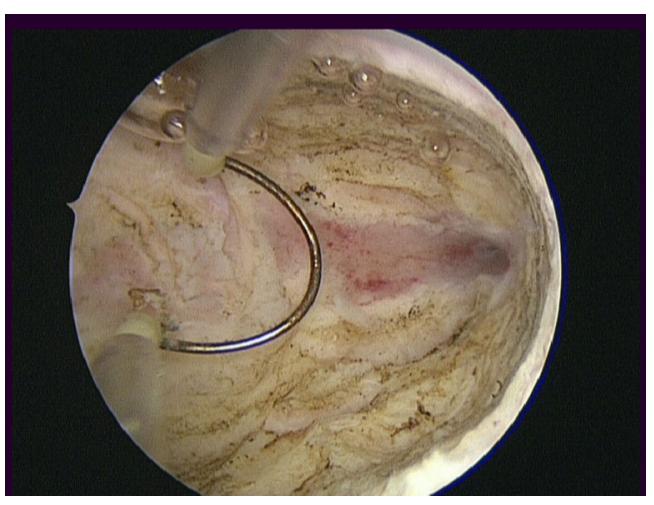

Fig. 1 Resection of the anterior cardinal strip of tissue followed by resection of the posterior and the two lateral cardinal strips without treating fundus and cornual areas 
Office hysteroscopy

Control hysteroscopy was scheduled at 3, 12, 24, and 60 months after the surgery. It was performed by using vaginoscopic approach, with a continuous-flow hysteroscope using Telescope $2.9 \mathrm{~mm}$ (HOPKINS II Forward-Oblique Telescope $30^{\circ}$; Karl Storz, Tuttlingen, Germany) [17, 18]. The uterine cavity was distended with temperate saline solution and irrigated using an electronic irrigation pump (Hysteromat, Karl Storz ${ }^{\circledR}$, Karl Storz, Tuttlingen, Germany). Examination was performed in an office setting, without anesthesia or cervical dilatation.

Main outcomes of the study

Outcome measures referred to changes in bleeding patterns, safeness, and patients' acceptability. In details, after modified TCER, women underwent office hysteroscopy for uterine cavity evaluation (with endometrial biopsy) 3, 12, 24, and 60 months after TCER, and simultaneously, patients were asked about the amelioration or persistence of bleeding, duration of amenorrhea, improvement of dysmenorrhea, and if they need any hormonal or surgical treatment for heavy bleeding after modified TCER.

Modified TCER was considered successful when it was associated with amenorrhea, hypomenorrhea, and eumenorrhea. A women was defined as amenorrheic when reporting persistent ceasing of menstruation after surgery. Eumenorrhea referred to regular menstrual cycle with average length of 28 days (range, 21-35 days), lasting on average for 4 days (range, 1-8 days) and of normal quantity (flowing less than $80 \mathrm{~mL}$ per cycle). Hypomenorrhea referred to menstruations regular in frequency but poor in quantity and/or lasting less than 2 days [1]. Resection was considered non-effective when the patient reported persistence or relapse of menorrhagia.

Interviews were done at the time of every follow-up hysteroscopy, and data were related to patients' subjective experiences related/consequent to modified TCER and their health status concerning endometrial status.

\section{Statistical analysis}

All data were analyzed with Prism software (GraphPad Software Inc., San Diego, CA, USA) and expressed as mean $\pm \mathrm{SD}$. The Kolmogorov-Smirnov test was used to evaluate whether values had a Gaussian distribution, in order to choose between parametric and non-parametric statistical tests. Therefore, the unpaired $t$ test was used to compute statistical significance, and $\chi$-square and Fisher exact test to analyze differences between proportions. Statistical significance was assumed for values of $P<0.05$.

\section{Results}

Some patients were not included in the study. Exclusion criteria were the following: not confirmed diagnosis of menorrhagia ( 26 women), uterine size $>12 \mathrm{~cm}$ (10 patients), presence of large organic intrauterine lesions (endometrial polyp $>3 \mathrm{~cm}$, submucous myomas $\mathrm{G} 0>2 \mathrm{~cm}$, and submucous myomas G1 and G2) (15 patients); desire of future pregnancy (eight patients), cervical, and endometrial pre- and malignant conditions or adnexal pathologies (10 patients).

Eighty-four patients out of 153 (age $45.37 \pm 4.02$ ) entered the study. The clinical study protocol consisted of modified TCER, followed by the assessment of endometrial cavity by office diagnostic hysteroscopy $3,12,24$, and 60 months after the surgery. Table 1 shows clinical and demographic details of the population evaluated in the study. Endometrial resection was successfully performed in all patients enrolled. No one of the patients had early or late complications. In 24 out of 84 patients, endometrial resection was associated with the simultaneous removal of small polyps $(n=15)$ or myomas $(n=9)$ (data not shown). In any case, the time required for endometrial resection with associated polypectomy $(13.31 \pm 6.09 \mathrm{~min})$ or myomectomy $(14.7 \pm 8.2 \mathrm{~min})$ did not differ when compared to the endometrial resection alone $(11.91 \pm 4.15 \mathrm{~min}$; $P>0.05$ ), as well as the time spent for cervical dilatation and the infusion volume needed for uterine distension (data not shown). In 11 cases (13\%), adenomyosis was evidenced by histopathologic examination.

Follow-up outcomes: clinical findings

Eleven women dropped out (13.1\%) and did not complete the first year of follow-up, while $73(86.9 \%)$ patients completed the follow-up for at least 60 months (Table 2) and 61 (83.6\%) of these reached a 84-month follow-up. During the observational time interval, bleeding patterns were observed in eumenorrhea in 50 out of 73 women $(68.5 \%)$, hypomenorrhea in four patients $(5.5 \%)$, and amenorrhea in 10 subjects $(13.7 \%)$ (Table 2$)$. None of them reported spotting, neither dysmenorrhea onset or worsening, nor medium- $/$

Table 1 Anthropometric and surgical data related to the population prospectively evaluated

\begin{tabular}{lc}
\hline Age (years) & $45.37 \pm 4.02$ \\
Gravida & $2.3 \pm 1.2$ \\
Parity & $2.4 \pm 1.2$ \\
BMI $\left(\mathrm{kg} / \mathrm{m}^{2}\right)$ & $28.7 \pm 3.3$ \\
Mean operating time (min) & $12.4 \pm 1.8$ \\
Cervical dilatation (min) & $1.4 \pm 0.4$ \\
Infusion volume (mL) & $2,500 \pm 550$ \\
Fluid deficit $(\mathrm{mL})$ & $250 \pm 110$ \\
\hline
\end{tabular}

Data are reported as mean $\pm \mathrm{SD}$ 
Table 2 Data related to the clinical findings retrieved at follow-up after modified TCER in women prospectively evaluated

\begin{tabular}{ll}
\hline Patients underwent MTCER $(n ; \%)$ & $84(100 \%)$ \\
Follow-up completed after 3 months $(n ; \%)$ & $84(100)$ \\
Follow-up completed after 60 months $(n ; \%)$ & $73(86.9)$ \\
Bleeding patterns after TCER & \\
Eumenorrhea $(n ; \%)$ & $50 / 73(68.5)$ \\
Hypomenorrhea $(n ; \%)$ & $4 / 73(5.5)$ \\
Amenorrhea $(n ; \%)$ & $10 / 73(13.7)$ \\
Menorrhagia $(n ; \%)$ & $7 / 73(9.6)$ \\
Recurrence of AUB/DUB $(n ; \%)$ & $2 / 73(2.7)$ \\
\hline
\end{tabular}

long-term complications such as pregnancy or complications putatively related to the development of intra-cavitary adhesions, such as hematometra and/or cornual hematometra, as observed at diagnostic office hysteroscopy.

Seven $(9.6 \%)$ patients continued to have menorrhagia, and among them, two of the 11 cases were with adenomyosis $(18.2 \%)$. Three patients underwent laparoscopic hysterectomy $(n=3)$ after an average interval of 6 months (range, $1-11$ months), two patients underwent hormonal treatment by means of levonorgestrel-based intrauterine device, and the remaining two women underwent 4-month GnRH analog administration $(n=2)$ (data not shown). In these patients, a second modified TCER was not performed because patients refused such a type of treatment.

In addition, AUB recurred in two women (2.7 \%) after 12 and 60 months after endometrial resection (Table 2). They underwent a new modified TCER or laparoscopic hysterectomy (for the women in whom recurrence occurred 60 months after modified TCER) (data not shown).

\section{Follow-up outcome: data from interview}

At the end of 3-month follow-up, 63 women (86.3\%) stated they were satisfied by the surgery so much that they would recommend it to women with menorrhagia, whereas the remaining 10 patients $(13.7 \%)$ would not, for reasons mainly related to the fear of general anesthesia $(n=8 ; 80.0 \%)$ or office hysteroscopy in the follow-up $(n=2 ; 20 \%)$. None of the patients had symptoms or conditions invalidating and/or limiting their quality of life (data not shown).

Follow-up outcome: hysteroscopic findings

Considering findings recorded at hysteroscopic follow-up, none of the patients was found to have intra-uterine adhesions or cavity contracture after 3 months. It was possible to evaluate the entire cavity, including the cornual area and the tubal ostia in all patients (Fig. 2). The same findings were obtained

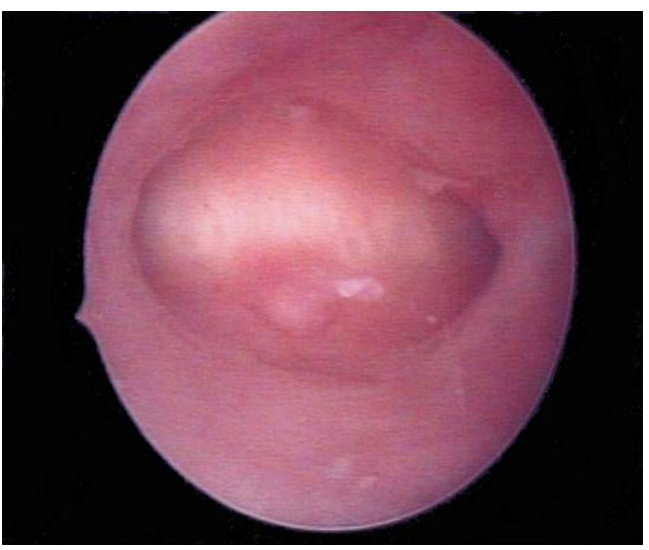

Fig. 2 Hysteroscopic appearance of endometrial cavity at 3-month follow-up after MTCER. None of the patients were found to have intrauterine adhesions

at all time points in the follow-up, even if the uterine cavity at the hysteroscopic evaluation performed after 12,24 , and 60 months from endometrial resection was markedly reduced to a narrow tube as a result of fibrosis and contracture (Fig. 3). In any case, the entire uterine cavity, including the cornual areas, was found to be open and the tubal ostia were visualized at all time points of follow-up. In addition, histological evaluation of endometrial biopsy annually performed failed to found cancerous or pre-cancerous endometrial lesions (data not shown).

\section{Discussion}

The present study first refers on the clinical efficiency and patients' perception of a new endometrial resection technique, by using which menorrhagia was resolved in the majority of patients, without surgical complications, no intrauterine adhesions formation in the follow-up, no fluid overload syndrome, short operative time, and a high degree of patients' satisfaction.

The reasons that led us to devise such a new technique are related to the fact that the conventional hysteroscopic TCER is

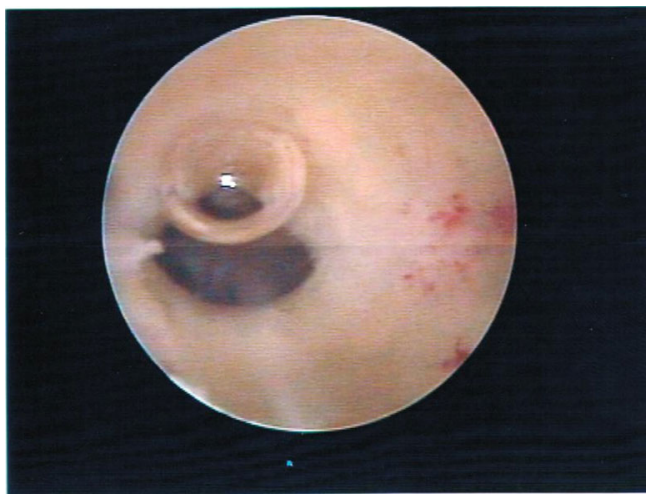

Fig. 3 A narrowing of the cavity is evidenced at 60 months but it does not hinder to evaluate the entire cavity, including the cornual area 
sometime associated to clinical problems, like surgical (perforation of the uterus), vascular complications or fluid overload syndrome [5-12]. Moreover, like resectoscopic myomectomy, TCER is a surgical procedure suggested only to experienced surgeons [19]. Consequently, our aim was to simplify and to accelerate the procedure maintaining the success rate and possibly decreasing the complications in order to make it accessible even to less experienced gynecologists. In addition, we intended to decrease the occurrence of intrauterine adhesions, contractures, and/or hematometra [12, 20-22]. These last side effects are due to the fact that after the endometrium is destroyed or resected, the myometrium is exposed and intrauterine walls, collapsing on each other, have a natural tendency to grow together. The final result is the intrauterine contracture and marked reduction of the endometrial cavity to a narrow tubular structure as a result of fibrosis that often obstructs the cornual area. This mechanism is responsible for the occurrence of synechiae in $40 \%$ of women submitted to total TCER [2] that may limit the access to the uterine cavity, hematometra, that usually localizes in the fundus of the uterus, or obstruction of the cornual area in $13 \%$ of cases $[11,22,23]$. The persistence in these obstructed areas of islands of endometrial tissue may cause retrograde menstruation or symptomatic cornual hematometra, with an incidence of even $18 \%$, causing painful distention of the uterus [24]. The potential of all these complications may limit the clinical efficacy of hysteroscopic TCER and can require hysterectomy. In our technique, the resection of the endometrium and of the first myometrial layers was limited only on the anterior and posterior and lateral walls, without treating fundal and cornual areas by using the rollerball, as usually performed [1, 2] The initial hypothesis was that preserving endometrial mucosa of the fundal and cornual areas could decrease the risk of synechiae or focal hematometra facilitating the longterm uterine inspection $[15,25]$. The possibility that this approach could result in menorrhagia or bleeding persistence due to the endometrial mucosa not removed was not shown by our study. Moreover, in our case series, we failed to detect intrauterine adhesions at all evaluated time points after 12, 24, and 60 months and it was possible to carry out an endometrial biopsy sampling for histological evaluation despite the presence of some cavity contracture in the long-term follow-up. As possible explanation, one may propose that the integrity of corneal and fundal areas might sustain intrauterine walls.

On this regard, the work of McCausland and McCausland was pioneering, since recommending ablation of only one wall of the uterine cavity and avoiding the cornual areas reduced the incidence of adhesions formation after TCER [26]. Moreover, other authors have already proposed the resection of the entire upper uterine fundus, but sparing the isthmus and the immediate supraisthmic region to prevent hematometra caused by stenosis at the level of the cervical isthmus [27].
Findings obtained in the long-term follow-up of the present study showed the absence of intrauterine adhesions and/or hematometra: no patient reported, both in the hysteroscopic and clinical verification, the appearance or worsening of dysmenorrhea, and none of them had long-term complications ascribable to the development of intrauterine synechiae such as cornual hematometra. These findings lead us to suggest that the modified TCER we are proposing is able to avoid the formation of synechiae or shrinkage of the uterine cavity.

The second clinical matter that merits discussion refers to the absence in our study of intra- and peri-operative complications (uterine perforation, hemorrhage, excess fluid absorption, and thermic damages to peri-uterine structures). Cornual myometrium is indeed notoriously thin and thus with low resistance, therefore representing the critical area for any procedure performed at uterine fundus. Deciding not to treat such zone, we simplified the procedure making uterine perforations or thermic damages to peri-uterine structures improbable or at least less frequent compared to the conventional technique. Nevertheless, in modified TCER, the exposure of a smaller surface of the myometrium and the short operating time needed may together contribute to reduce absorption of hypotonic, electrolyte-free non-conductive distention solution, consequently not allowing the development of the overload syndrome [28].

It could be criticized that the residual endometrial tissue not removed in uterine fundus and cornual areas may be the site of putative pre- or markedly malignant lesions. We took care of this criticism, and so, we submitted our patients to an endometrial surveillance by endometrial biopsy under hysteroscopic guidance. Our follow-up hysteroscopies consented to visualize and collect samples from fundus and corneal areas in every patient. On the contrary, in the conventional TCER technique, the habitual collapse of the uterine walls and the formation of synechiae may hinder endometrial biopsy. Whether such a problem does not seem to affect low-risk population (i.e., patients with pre-ablation biopsy negative for hyperplasia and negative medical history for common risk factors for uterine neoplasia) [29], modified TCER may represent a valid therapeutic option for those patients considered at increased risk of developing hyperplasia and endometrial carcinoma. The unquestionable advantage of TCER, as opposed to new-generation destructive ablation methods, is to provide additional tissue for histological examination of the endometrium so that it is possible to detect any presence of micro foci of neoplasia or a high risk for it in the resected material previously not diagnosed in pre-surgical biopsy [30]. On the other hand, patients with increased risk of hyperplasia or endometrial neoplasia frequently are also at higher risk for major surgery, such is hysterectomy. Indeed, cardiovascular diseases, severe obesity, chronic nephropathies, coagulopathies, and hepatopathies are often co-existent with a history 
of meno- or metrorrhagia and also imply a high surgical and anesthesiological risks. The modified TCER we propose can be even more suitable for those "complex" patients, since it would have the advantage to be performed more quickly, with less intra- and peri-operative complications that the endometrial resection used so far.

In conclusion, despite the limitation due to the small sample size, our data on complications and the low (11.3\%) prevalence of bleeding persistence after operative hysteroscopy would suggest that modified TCER is a technique easy to perform, effective in the resolution of long-term menorrhagia and useful in patients with high surgical and anesthesiological risks. In addition, this new approach allows avoiding the formation of synechiae and the shrinkage of the uterine cavity that may be the cause for various long-term complications, such as the delay in the diagnosis of endometrial carcinoma onset.

Informed consent All procedures followed were in accordance with the ethical standards of the responsible committee on human experimentation (institutional and national) and with the Helsinki Declaration of 1975 , as revised in 2000. Informed consent was obtained from all patients for being included in the study.

Conflict of interest Pietro Litta, Luigi Nappi, Pasquale Florio, Luca Mencaglia, Mario Franchini, and Stefano Angioni declare that they have no conflict of interest.

\section{References}

1. Magos AL, Baumann R, Lockwood GM, Turnbull AC (1991) Experience with the first 250 endometrial resection for menorrhagia. Lancet 337:1074-1078

2. Wortman M, Daggett A (1994) Hysteroscopic endomyometrial resection: a new technique for the treatment of menorrhagia. Obstet Gynecol 83:295-299

3. Farquhar CM, Steiner CA (2002) Hysterectomy rates in the United States 1990-1997. Obstet Gynecol 99:229-234

4. Vilos GA, Harding PG, Ettler HC (2002) Resectoscopic surgery in women with abnormal uterine bleeding and nonatypical endometrial hyperplasia. J Am Assoc Gynecol Laparosc 9:131-137

5. Lethaby A, Penninx J, Hickey M, Garry R, Marjoribanks J (2013) Endometrial resection and ablation techniques for heavy menstrual bleeding. Cochrane Database Syst Rev 8, CD001501

6. Litta P, Merlin F, Pozzan C, Nardelli GB, Capobianco G, Dessole S, Ambrosini A (2006) Transcervical endometrial resection in women with menorrhagia: long-term follow-up. Eur J Obstet Gynecol Reprod Biol 125:99-102

7. MacLean-Fraser E, Penava D, Vilos GA (2002) Perioperative complication rates of primary and repeat hysteroscopic endometrial ablations. J Am Assoc Gynecol Laparosc 9:175-177

8. Papadopoulos NP, Magos A (2007) First-generation endometrial ablation: roller-ball vs loop vs laser. Best Pract Res Clin Obstet Gynaecol 21(6):915-929

9. Paschopoulos M, Polyzos NP, Lavasidis LG, Vrekoussis T, Dalkalitsis N, Paraskevaidis E (2006) Safety issues of hysteroscopic surgery. Ann N Y Acad Sci 1092:229-234
10. Perino A, Castelli A, Cucinella G, Biondo A, Pane A, Venezia R (2004) A randomized comparison of endometrial laser intrauterine thermotherapy and hysteroscopic endometrial resection. Fertil Steril 82(3):731-734

11. Boujida VH, Philipsen T, Pelle J, Joergensen JC (2002) Five-year follow-up of endometrial ablation: endometrial coagulation versus endometrial resection. Obstet Gynecol 99(6):988-992

12. Propst AM, Liberman RF, Harlow BL, Ginsburg ES (2000) Complications of hysteroscopic surgery: predicting patients at risk. Obstet Gynecol 96(4):517-520

13. Higham JM, O'Brian PMS, Shaw RW (1990) Assessment of menstrual blood loss using a pictorial chart. Br J Obstet Gynaecol 97: 734-739

14. De Angelis C, Carnevale A, Santoro G, Nofroni I, Spinelli M, Guida M, Mencaglia L, Di Spiezio Sardo A (2013) Hysteroscopic findings in women with menorrhagia. J Minim Invasive Gynecol 20(2):209214

15. Angioni S, Loddo A, Milano F, Piras B, Minerba L, Melis GB (2008) Detection of benign intracavitary lesions in postmenopausal women with AUB. A prospective study on outpatients hysteroscopy and blind biopsies. J Minim Invasive Gynecol 15(1):87-91

16. Florio P, Puzzutiello R, Filippeschi M, D’Onofrio P, Mereu L, Morelli R, Marianello D, Litta P, Mencaglia L, Petraglia F (2012) Low-dose spinal anesthesia with hyperbaric bupivacaine with intrathecal fentanyl for operative hysteroscopy: a case series study. J Minim Invasive Gynecol 19(1):107-112

17. Di Spiezio Sardo A, Bettocchi S, Spinelli M, Guida M, Nappi L, Angioni S, Sosa Fernandez LM, Nappi C (2010) Review of new office-based hysteroscopic procedures 2003-2009. J Minim Invasive Gynecol 17:436-448

18. Daniilidis A, Pantelis A, Dinas K, Tantanasis T, Loufopoulos PD, Angioni S, Carcea F (2012) Indications of diagnostic hysteroscopy, a brief review of the literature. Gynecol Surg 9(1):23-28

19. Litta P, Conte L, De Marchi F, Saccardi C, Angioni S (2014) Pregnancy outcome after hysteroscopic myomectomy. Gynecol Endocrinol 30(2):149-152

20. Overton C, Hargreaves J, Maresh M (1997) A national survey of the complications of endometrial destruction for menstrual disorders: the MISTLETOE study. Minimally Invasive Surgical Techniques-Laser, EndoThermal or Endoresection. Br J Obstet Gynaecol 104(12): 1351-1359

21. Hart R, Magos A (1997) Endometrial ablation. Curr Opin Obstet Gynecol 9(4):226-232

22. Wortman M, Daggett A (2001) Reoperative hysteroscopic surgery in the management of patients who fail endometrial ablation and resection. J Am Assoc Gynecol Laparosc 8(2): 272-277

23. Tapper AM, Heinonen PK (1995) Hysteroscopic endomyometrial resection for the treatment of menorrhagia-follow-up of 86 cases. Eur J Obstet Gynecol Reprod Biol 62(1):75-79

24. McCausland AM, McCausland VM (2002) Frequency of symptomatic cornual hematometra and postablation tubal sterilization syndrome after total rollerball endometrial ablation: a 10-year followup. Am J Obstet Gynecol 186:1274-1280

25. Litta P, Merlin F, Saccardi C, Pozzan C, Sacco G, Fracas M, Capobianco G, Dessole S (2005) Role of hysteroscopy with endometrial biopsy to rule out endometrial cancer in postmenopausal women with abnormal uterine bleeding. Maturitas 50(2): $117-123$

26. McCausland AM, McCausland VM (1999) Partial rollerball endometrial ablation: a modification of total ablation to treat menorrhagia without causing complications from intrauterine adhesions. Am J Obstet Gynecol 180:1512-1521

27. Perino A, Cittadini E, Colacurci N, De Placido G, Hamou J (1990) Endometrial ablation: principles and technique. Acta Eur Fertil 21(6): 313-317 
28. Witz CA, Silverberg KM, Burns WN, Schenken RS, Olive DL (1993) Complications associated with the absorption of hysteroscopic fluid media. Fertil Steril 60(5):745-756

29. Neuwirth RS, Loffer FD, Trenhaile T, Levin B (2004) The incidence of endometrial cancer after endometrial ablation in a low-risk population. J Am Assoc Gynecol Laparosc 11(4): 492-494

30. Ahonkallio SJ, Liakka AK, Martikainen HK, Santala MJ (2009) Feasibility of endometrial assessment after thermal ablation. Eur J Obstet Gynecol Reprod Biol 147(1):69-71 